\title{
Accurate DOA Estimations Using Microstrip Adaptive Arrays in the Presence of Mutual Coupling Effect
}

\author{
Qiulin Huang, Hongxing Zhou, Jianhui Bao, and Xiaowei Shi \\ Science and Technology on Antenna and Microwave Laboratory, Xidian University, Xian 710071, China \\ Correspondence should be addressed to Qiulin Huang; qiulhuang@mail.xidian.edu.cn
}

Received 30 August 2013; Accepted 24 October 2013

Academic Editor: Hon Tat Hui

Copyright (C) 2013 Qiulin Huang et al. This is an open access article distributed under the Creative Commons Attribution License, which permits unrestricted use, distribution, and reproduction in any medium, provided the original work is properly cited.

\begin{abstract}
A new mutual coupling calibration method is proposed for adaptive antenna arrays and is employed in the DOA estimations to calibrate the received signals. The new method is developed via the transformation between the embedded element patterns and the isolated element patterns. The new method is characterized by the wide adaptability of element structures such as dipole arrays and microstrip arrays. Additionally, the new method is suitable not only for the linear polarization but also for the circular polarization. It is shown that accurate calibration of the mutual coupling can be obtained for the incident signals in the $3 \mathrm{~dB}$ beam width and the wider angle range, and, consequently, accurate [1D] and [2D] DOA estimations can be obtained. Effectiveness of the new calibration method is verified by a linearly polarized microstrip ULA, a circularly polarized microstrip ULA, and a circularly polarized microstrip UCA.
\end{abstract}

\section{Introduction}

In the last several decades, many direction-of-arrival (DOA) estimation algorithms with superresolution have been proposed, such as the multiple signal classification (MUSIC), the estimations of signal parameters via rotational invariance techniques (ESPRIT), the maximum likelihood (ML), and the subspace fitting (SF) [1-4]. Employing the ideal array manifold, these algorithms can provide excellent DOA estimation performances. For the actual antenna array, however, the array manifold cannot be regarded as that of the ideal point source array. The magnitude and phase of the received array signals are disturbed by the mutual coupling effect of the actual array, which would degrade the performances of the DOA estimation algorithms [5].

Many interests have been focused on the DOA estimations in the presence of mutual coupling effect over the past years. In order to obtain accurate DOA estimations, DOA estimation methods employing the actual array manifold were proposed [6-8]. Essentially, the actual manifold is equivalent to the array pattern. However, great effort is required to calculate and measure the actual manifold. Additionally, mass storage is needed for the manifold data in these methods. The transformation relationship between the actual array manifold and the array manifold of the ideal point source was proposed to decrease the storage of the manifold data $[9,10]$. In this method, a distortion matrix was employed to carry out the transformation with assuming that the distortion matrix is angular independent. The validity of this method was verified in the $1 \mathrm{D}$ DOA estimations using the antenna array composed of the collinear dipoles. However, for the antenna array that is composed of microstrip elements, this method is valid only in a very limited angle range. In the literature, the universal steering vector was used in DOA estimations employing the antenna array with arbitrary geometry [11]. In this method, the received signal can be used directly to carry out the DOA estimations without calibration. However, the universal steering vector is dependent on the incident angles and the polarization of the incoming signals.

In addition to the DOA estimation methods in the presence of the mutual coupling effect, the mutual coupling calibration methods are also employed in the DOA estimations. In these methods, the calibration matrix was obtained through various approaches, such as the open-circuit voltage method, the receiving mutual impedance method, and the 
minimum-norm method $[5,12,13]$. The research in [14] investigated the effects of ground parameters on the mutual impedance for DOA estimations, and some suggestions were put forward to improve the DOA estimations. In the opencircuit voltage method, the open-circuit voltages of the array were treated as the decoupled voltages [5]. For the antenna array composed of wire elements, accurate DOA estimations can be obtained by adopting the open circuit voltage method. For the antenna array composed of planar elements such as the microstrip antenna, however, the open-circuit voltage method would not take the positive role in the mutual coupling calibration owing to the strong open scattering effect. By contrast, the receiving mutual impedance method and the minimum-norm method can provide better calibration of the mutual coupling effect owing to consideration of the secondary scattering effect of the array. However, these methods are more suitable for the antenna array composed of wire elements, such as dipoles and monopoles.

In the research of the mutual coupling calibration, joint estimation methods of the DOAs and calibration matrix were also proposed [15-18]. This kind of methods utilized the array processing to obtain the DOAs and the calibration matrix simultaneously and can be employed in the arrays composed of various element geometries. These methods were usually used in the array with regular array structures, such as the uniform linear arrays (ULAs) and the uniform circular arrays (UCAs). For regular arrays, the number of mutual coupling parameters can be decreased owing to the regular array structure, which would benefit the joint estimations of the DOAs and calibration matrix. Presently, the time consumption to find the DOAs and calibration matrix is not acceptable for many actual applications, and many efforts have been made to decrease the complexity of the joint estimation algorithms.

In this paper, the element pattern reconstruction method is proposed to calibrate the mutual coupling effect. In this method, the calibration matrix is developed through the transformation relationship between the embedded element patterns and the isolated element patterns. The new method is based on the fact that when all of the embedded element patterns are transformed to coincide with the corresponding isolated element patterns in a certain direction, the received signals owing to this direction would be decoupled after being transformed with the same means. This method is characterized by the wide adaptability of element structures. It can be used in microstrip antenna arrays to obtain highly accurate calibration of the mutual coupling effect. In general, it can be done to reconstruct the element patterns of an array to coincide with those in the isolated state in the $3 \mathrm{~dB}$ beam width or a little wider angle range. This means that the calibration method can calibrate the incident signals coming from the angle range and provide necessary conditions for accurate DOA estimations. In order to testify the performance of the new calibration method, two microstrip arrays including a linearly polarized microstrip array and a circularly polarized microstrip array are designed to carry out the DOA estimations. In the numerical examples, the classical MUSIC algorithm is employed to find the directions of the incident signals.

\section{Theory}

Consider an antenna array composed of $N$ elements; each element is terminated with the load $Z_{L}$. In the development of the new calibration method, the main polarization component of the electric field of the array element is used since the main polarization component takes the dominant part in the total electric field of the array. For the linearly polarized array, the main polarization component is the $\theta$ component or the $\varphi$ component. For the circularly polarized array, it is the lefthand circular polarization (LHCP) component or the righthand circular polarization (RHCP) component. According to the basis of the new method, (1) is used to realize the transformation of the element patterns. Consider

$$
\left[\begin{array}{c}
E_{1}^{i}(\theta, \varphi) \\
E_{2}^{i}(\theta, \varphi) \\
\vdots \\
E_{N}^{i}(\theta, \varphi)
\end{array}\right]=\mathbf{C}\left[\begin{array}{c}
E_{1}(\theta, \varphi) \\
E_{2}(\theta, \varphi) \\
\vdots \\
E_{N}(\theta, \varphi)
\end{array}\right],
$$

where $E_{n}^{i}(\theta, \varphi)$ and $E_{n}(\theta, \varphi)$ for element $n$ represent the electric field of the isolated element and that of the embedded element, respectively. It is to be noted that the embedded element pattern for each element should be calculated with each element terminated with a load $Z_{L}$. The calibration matrix $\mathbf{C}$ has the dimension of $N \times N$. In order to solve the matrix $\mathbf{C}$, some directions should be sampled from all element patterns in (1). And then (1) is transformed into a solvable matrix function as follows:

$$
\begin{gathered}
{\left[\begin{array}{c}
E_{1}^{i}\left(\theta_{1}, \varphi_{1}\right) \cdots E_{1}^{i}\left(\theta_{M}, \varphi_{M}\right) \\
E_{2}^{i}\left(\theta_{1}, \varphi_{1}\right) \cdots E_{2}^{i}\left(\theta_{M}, \varphi_{M}\right) \\
\vdots \\
E_{N}^{i}\left(\theta_{1}, \varphi_{1}\right) \cdots E_{N}^{i}\left(\theta_{M}, \varphi_{M}\right)
\end{array}\right]} \\
=\mathbf{C}\left[\begin{array}{c}
E_{1}\left(\theta_{1}, \varphi_{1}\right) \cdots E_{1}\left(\theta_{M}, \varphi_{M}\right) \\
E_{2}\left(\theta_{1}, \varphi_{1}\right) \cdots E_{2}\left(\theta_{M}, \varphi_{M}\right) \\
\vdots \\
E_{N}\left(\theta_{1}, \varphi_{1}\right) \cdots E_{N}\left(\theta_{M}, \varphi_{M}\right)
\end{array}\right] .
\end{gathered}
$$

Consequently, the least square solution that satisfies $\min _{\| \mathbf{C E}-\mathbf{E}^{i}||}\|\mathbf{C}\|$ can be obtained; that is,

$$
\mathbf{C}=\mathbf{E}^{i} \mathbf{E}^{H}\left(\mathbf{E E}^{H}\right)^{-1},
$$

where $\mathbf{E}$ and $\mathbf{E}^{i}$ represent the pattern matrix for all of the embedded elements and isolated elements, respectively. The operator $\|\cdot\|$ denotes the $F$-norm of a matrix. For the matrix C with the dimension of $N \times N$, the $F$-norm is written as

$$
\|\mathbf{C}\|=\left\{\sum_{n=1}^{N} \sum_{m=1}^{N}\left|c_{n m}\right|^{2}\right\}^{1 / 2},
$$

where $c_{n m}$ is an entry of the matrix $\mathbf{C}$. 
The electric fields in the previous equations can be calculated by various methods, such as the method of moments $(\mathrm{MOM})$, the finite-element method (FEM), and the finitedifference time domain (FDTD) method.

The matrix $\mathbf{C}$ calculated from (3) can be used to reconstruct the element patterns and to calibrate the mutual coupling effect of the array. Assume that the sample matrix of the received signals is $\mathbf{X}^{\prime}$ and can be written by

$$
\mathbf{X}^{\prime}=\mathbf{A S}+\mathbf{N}_{\mathbf{0}},
$$

where $\mathbf{A}$ represents the direction matrix, $\mathbf{S}$ represents the sampled stochastic data matrix, and $\mathbf{N}_{0}$ represents the add white Gaussian noise matrix. The calibrated sample matrix can be calculated by the following formula:

$$
\mathbf{X}=\mathbf{C X}^{\prime} .
$$

For the actual antenna array, the received signals influenced by the mutual coupling effect can be generated via the embedded element pattern matrix $\mathbf{E}$ which acts as the actual direction matrix. Another method to obtain the actual direction matrix is calculating the induced terminal voltage vectors when a plane wave impinges on the array in the same direction of the incident signal.

When the received signals are calibrated by the calibration matrix, they can be imported to the MUSIC algorithm to find the directions of the incident signals. The covariance matrix of the calibrated received signals $\mathbf{X}$ is written as

$$
\mathbf{R}_{X X}=E\left\{\mathbf{X X}^{H}\right\},
$$

where $E\{\cdot\}$ represents the statistical expectation and the superscript $H$ represents the complex conjugate transpose. Both the signal eigenvectors and the noise eigenvectors can be calculated by the previous covariance matrix. And then the orthogonal property between the signal eigenvectors and noise eigenvectors is employed to estimate the DOAs of the incident signals by searching the peaks of the MUSIC spectrum given by

$$
\begin{aligned}
& P_{\text {MUSIC }}(\theta, \varphi) \\
& =\frac{\mathbf{a}^{H}(\theta, \varphi) \mathbf{a}(\theta, \varphi)}{\left[\mathbf{a}^{H}(\theta, \varphi) \mathbf{U}_{N} \mathbf{U}_{N}^{H} \mathbf{a}^{H}(\theta, \varphi)\right]},
\end{aligned}
$$

where $\mathbf{U}_{N}$ is the matrix whose columns are the noise eigenvectors of the covariance matrix $\mathbf{R}_{X X}$ and $\mathbf{a}(\theta, \varphi)$ denotes the ideal steering vector of the antenna array.

In general, the new calibration method utilizes the relationship between the embedded element patterns and isolated element patterns. As is known, the received signal owing to the isolated element is not influenced by the mutual coupling effect. Consequently, accurate mutual coupling calibration can be provided by the new method. In Su's method that is proposed in [9], the relationship between the embedded element patterns and the patterns of ideal point source was employed. However, Su's method catered for the ideal point source model in the array processing, which would introduce extra mutual coupling effect. Of course, Su's method can
TABLE 1: Parameters of the incident signals.

\begin{tabular}{lccc}
\hline Incident signals & $\varphi$ & $\theta$ & SNR $(\mathrm{dB})$ \\
\hline Signal 1 & $-50^{\circ}$ & $90^{\circ}$ & 20 \\
Signal 2 & $-20^{\circ}$ & $90^{\circ}$ & 20 \\
Signal 3 & $0^{\circ}$ & $90^{\circ}$ & 20 \\
\hline
\end{tabular}

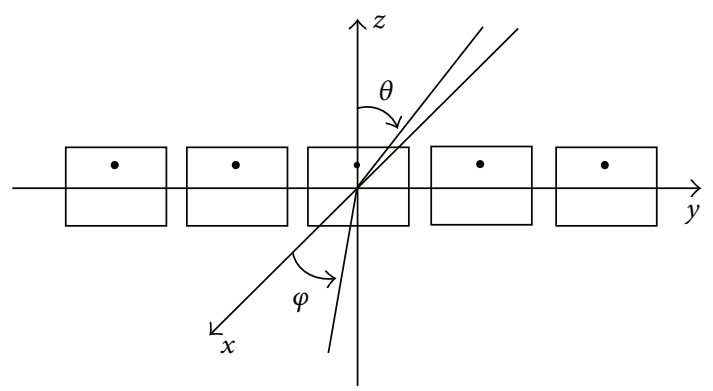

FIGURE 1: Structure of the linearly polarized microstrip array and the coordinate system.

provide good mutual coupling calibration for dipole or monopole arrays since the $\mathrm{H}$-plane pattern of the element is isotropic. By contrast, the new method can provide more accurate mutual coupling calibration, especially for arrays composed of planar elements. It can be seen from the following examples that the new method is suitable for the dipole array and the microstrip array and is effective for the linearly polarized array and the circularly polarized array.

\section{Numerical Examples}

In this section, a linearly polarized microstrip ULA, a circularly polarized microstrip ULA, and a circularly polarized microstrip UCA are employed to carry out the DOA estimations in the presence of mutual coupling effect. The two previous ULAs are used for one-dimensional DOA estimations, and the UCA is used for two-dimensional (2D) DOA estimations. The calibrated received signals are input to the MUSIC algorithm to find the directions of the incident signals, and the data sample is 1000 in the DOA estimations. Both arrays are composed of five elements, and they operate at the frequency of $3 \mathrm{GHz}$. Each element is terminated with the same load $Z_{L}=50 \Omega$. The microstrip arrays are fabricated on the substrate FR4 $\left(\varepsilon_{r}=4.4\right)$ with the thickness of $1.6 \mathrm{~mm}$. The EM simulation tool HFSS 13 is used to calculate the element patterns.

Three incident signals are involved in the following DOA estimations of (A) and (B). Parameters of all the incident signals are listed in Table 1.

(A) Linearly Polarized Microstrip ULA. The structure of the linearly polarized microstrip array with five elements is shown in Figure 1. The element spacing is $d=0.4 \lambda$. The length and width of the patch is $30.2 \mathrm{~mm}$ and $22.8 \mathrm{~mm}$, respectively. The feed point is set with the distance of $6.2 \mathrm{~mm}$ from the upper edge. The $3 \mathrm{~dB}$ beam width of the element is about $90^{\circ}$ in the xoy plane. The incident angle of Signal 1 is in 


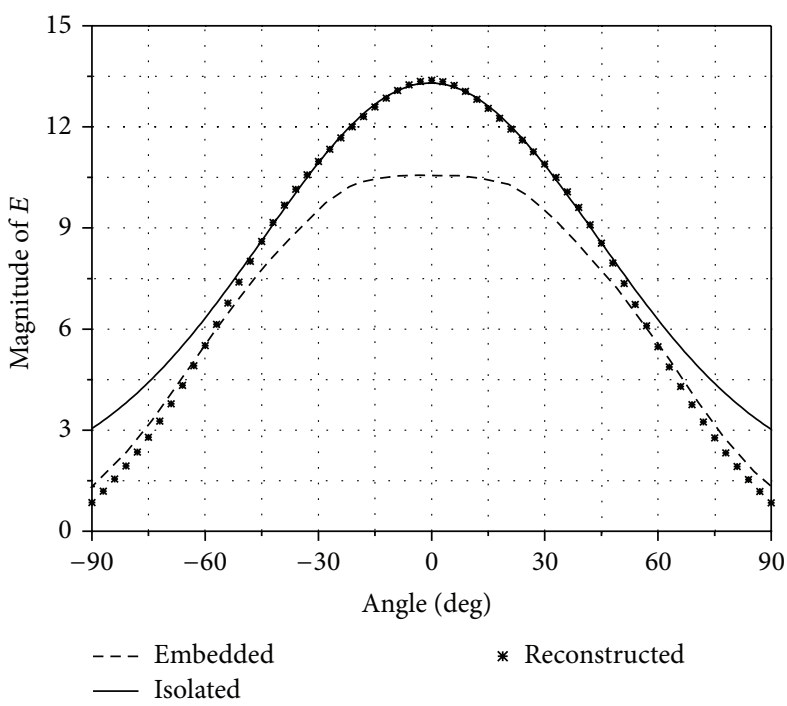

(a)

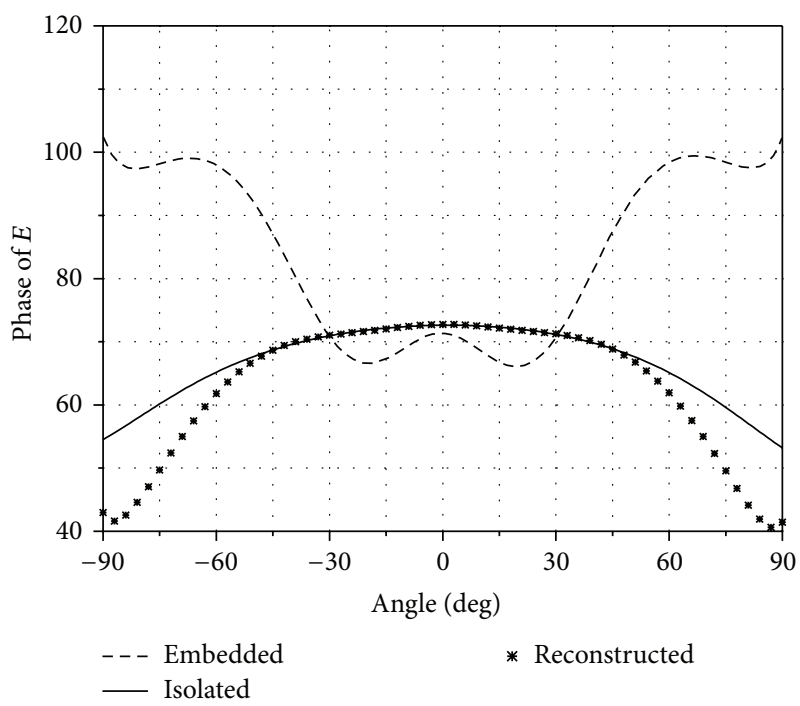

(b)

FIgURE 2: Magnitude and phase of $\theta$ component of the electric field in three cases for the linearly polarized microstrip array.

the outside of the $3 \mathrm{~dB}$ beam width, and the incident angles of the other two signals are in the $3 \mathrm{~dB}$ beam width. Assume that all incident signals are of the $z$-polarization. In the xoy plane, the main polarization component is the $\theta$ component or the $z$ component of the electric field. In order to calculate the calibration matrix via (3), 101 directions of the $\theta$ component of electric field are sampled from the angle range of $\left[-50^{\circ}\right.$, $\left.50^{\circ}\right]$ in the $x o y$ plane.

The calibration matrix is first used to reconstruct the element patterns of the array. The magnitude and phase of the central element in three states are shown in Figure 2. It can be seen that both the magnitude and the phase of the reconstructed element pattern of the element are coincident with those of the isolated element pattern in the angle range that is slightly larger than the $3 \mathrm{~dB}$ beam width. This means that the incident signals in this angle range can be calibrated with high accuracy, and, consequently, the accurate DOA estimations can be obtained, which can be seen from Figure 3 . The performance of the DOA estimations is seriously degraded by the mutual coupling effect of the array, and none of the DOAs of the incident signals can be found from the MUSIC spectrum without calibration. Large errors of the DOA estimations can be seen for all of the incident signals when the open-circuit voltage method is employed. For Su's method, the DOA estimation bias exists when the incident signals get far from the norm direction. However, the performance of the DOA estimations is significantly improved when the proposed method is used. More accurate DOA estimations are provided by the proposed method in the $3 \mathrm{~dB}$ beam width and the vicinity. The DOA estimation bias for the linearly polarized array can be found in Table 2 .

(B) Circularly Polarized Microstrip ULA. The circularly polarized microstrip element and array are shown in Figures 4 and 5, respectively. The element spacing is also $d=0.4 \lambda$. The $3 \mathrm{~dB}$ beam width of the element is about $100^{\circ}$
TABLE 2: DOA estimation bias for the linearly polarized array.

\begin{tabular}{lccc}
\hline & \multicolumn{3}{c}{ DOA estimation bias } \\
$\varphi$ & Open circuit & Su's method & Proposed method \\
\hline$-50^{\circ}$ & $0.5^{\circ}$ & $3.4^{\circ}$ & $0.2^{\circ}$ \\
$-20^{\circ}$ & $3.5^{\circ}$ & $1.4^{\circ}$ & $0^{\circ}$ \\
$0^{\circ}$ & $2.8^{\circ}$ & $0.6^{\circ}$ & $0.2^{\circ}$ \\
\hline
\end{tabular}

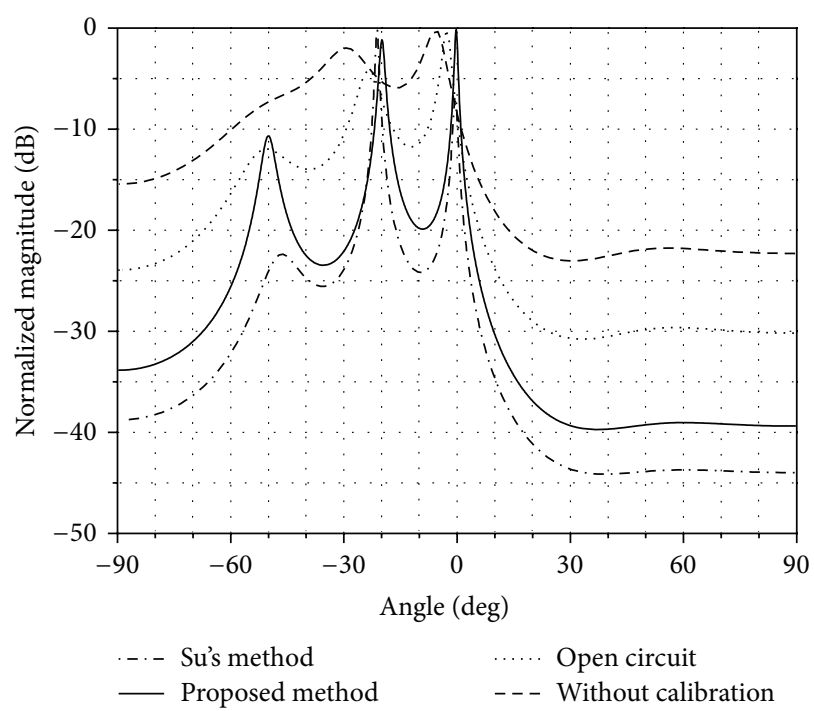

FIGURE 3: Spatial spectrum of DOA estimations for three incident signals employing the linearly polarized microstrip array.

in the xoy plane. It can be seen from Figure 6 that the RHCP component of the element pattern takes the dominant part in the total electric field. Therefore, the RHCP component of the electric field is employed to calculate the calibration matrix. Similar the example to previous, 101 directions of 
TABLE 3: DOA estimation bias for the circularly polarized array.

\begin{tabular}{lccc}
\hline & \multicolumn{3}{c}{ DOA estimation bias } \\
$\varphi$ & Open circuit & Su's method & Proposed method \\
\hline$-50^{\circ}$ & $4.6^{\circ}$ & $1.8^{\circ}$ & $0^{\circ}$ \\
$-20^{\circ}$ & $3^{\circ}$ & $1.5^{\circ}$ & $0.2^{\circ}$ \\
$0^{\circ}$ & $1.5^{\circ}$ & $0.6^{\circ}$ & $0.3^{\circ}$ \\
\hline
\end{tabular}

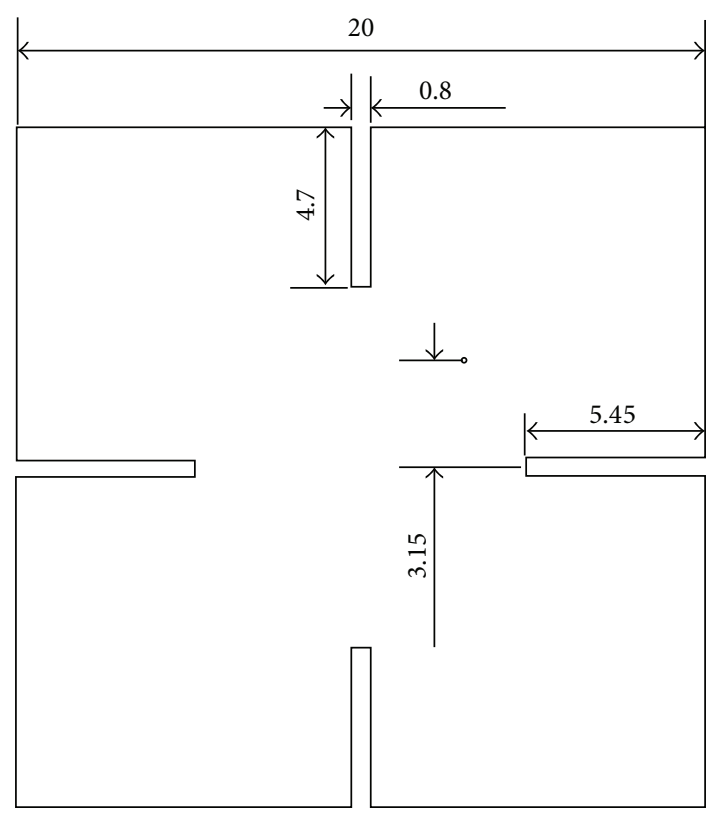

FIGURE 4: Profile of the circularly polarized microstrip element with the unit $\mathrm{mm}$.

the RHCP component of the electric field are chosen from the angle range of $\left[-50^{\circ}, 50^{\circ}\right]$ in the xoy plane to calculate the calibration matrix.

The reconstruction results of the embedded element pattern for the central element are shown in Figure 7. It can be seen that the coincidence between the reconstructed element pattern and the isolated element pattern is obtained in the angle range that is larger than $120^{\circ}$, which would bring about accurate DOA estimations in a wide angle range. The DOA estimations of three RHCP incident signals are given in Figure 8. It is shown that limited improvement of the performance of DOA estimations is provided by the open-circuit voltage method, and apparent DOA estimation errors exist for all of the three incident signals. The DOA estimation errors are decreased when Su's method is used. In contrast, more accurate DOA estimations for all incident signals could be obtained due to the element pattern reconstruction method. The DOA estimation bias for the circularly polarized array can be seen in Table 3 .

As seen from the pervious two numerical examples, proper calibration matrices can be obtained to carry out the mutual coupling calibration in the $3 \mathrm{~dB}$ beam width that is the main operating angle range of the array. And the generally, the coincidence between the reconstructed pattern and the isolated pattern is valid in the range beyond the $3 \mathrm{~dB}$ beam

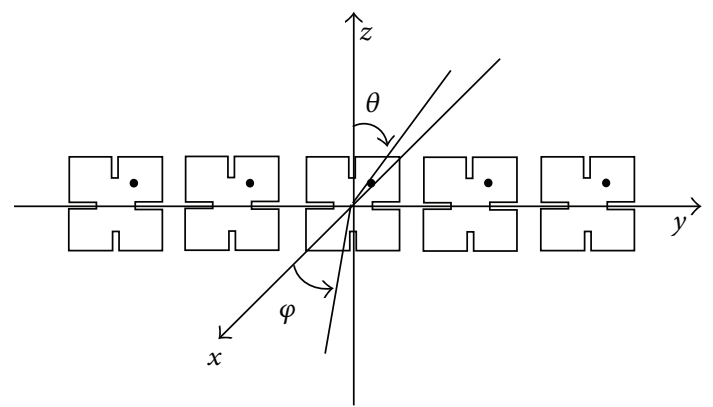

FIGURE 5: Structure of the circularly polarized microstrip array and the coordinate system.

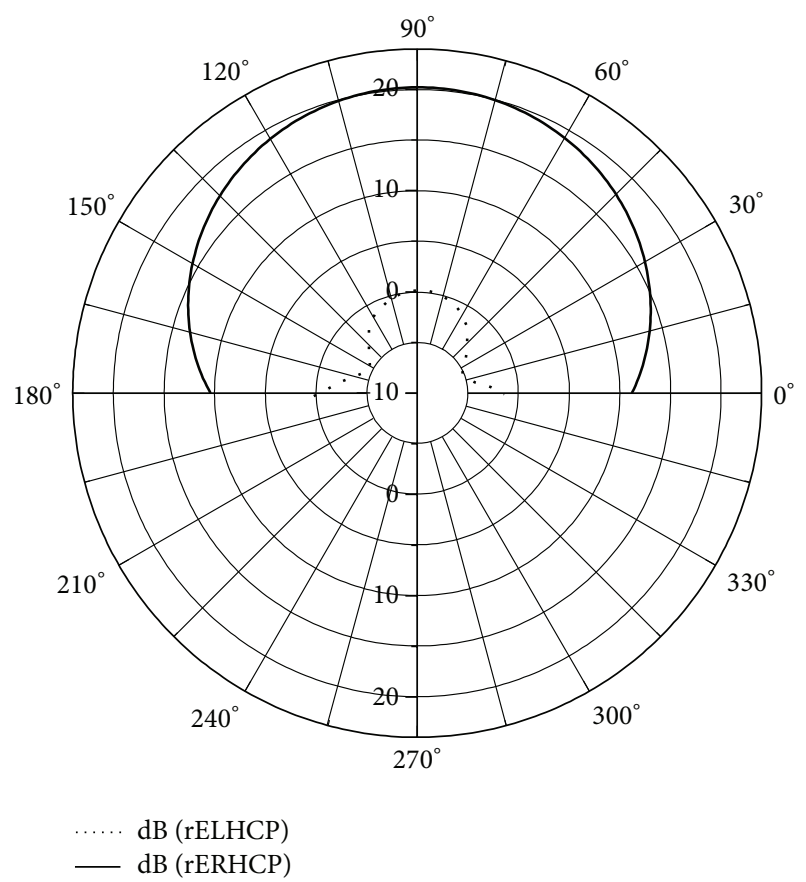

FIGURE 6: Isolated element pattern of the circularly polarized microstrip element.

width. Therefore, the microstrip element with larger $3 \mathrm{~dB}$ beam width would be more favorable to the mutual coupling calibrations. As for the polarization of elements, the less cross-polarization is more beneficial to the mutual coupling calibrations. It is quite understandable that the larger the main polarization component is, the less the difference between the theoretical calibration matrix and the calibration matrix actually required is.

(C) Circularly Polarized Microstrip UCA. The circularly polarized microstrip UCA is composed of six elements as given in Figure 4. The structure of the UCA with a radius of $0.5 \lambda$ is shown in Figure 9. It is designed for the 2D DOA estimations. Two incident signals are considered and the correlated parameters are listed in Table 4. In order to obtain a valid calibration matrix for $2 \mathrm{D}$ DOA estimations, enough direction samples are chosen in the main operating angular 


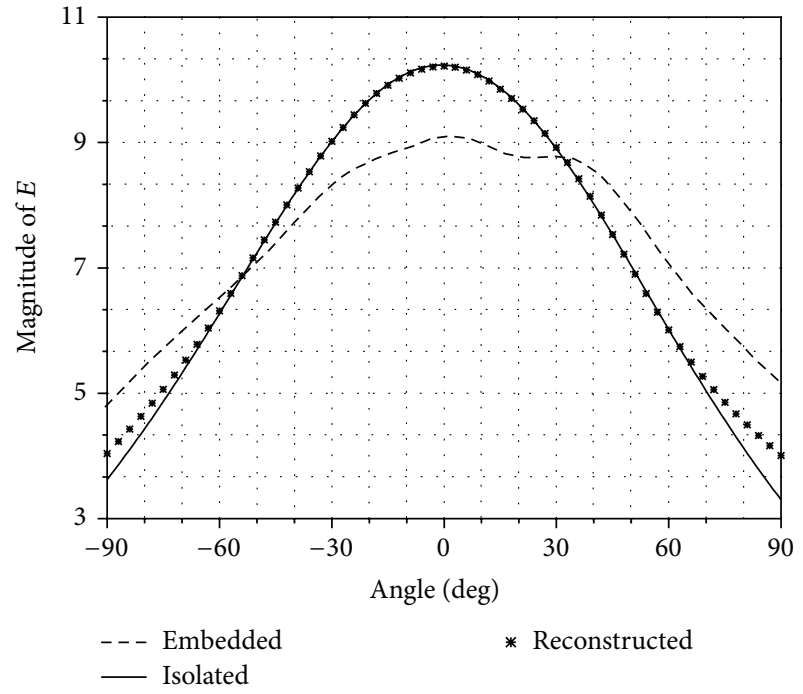

(a)

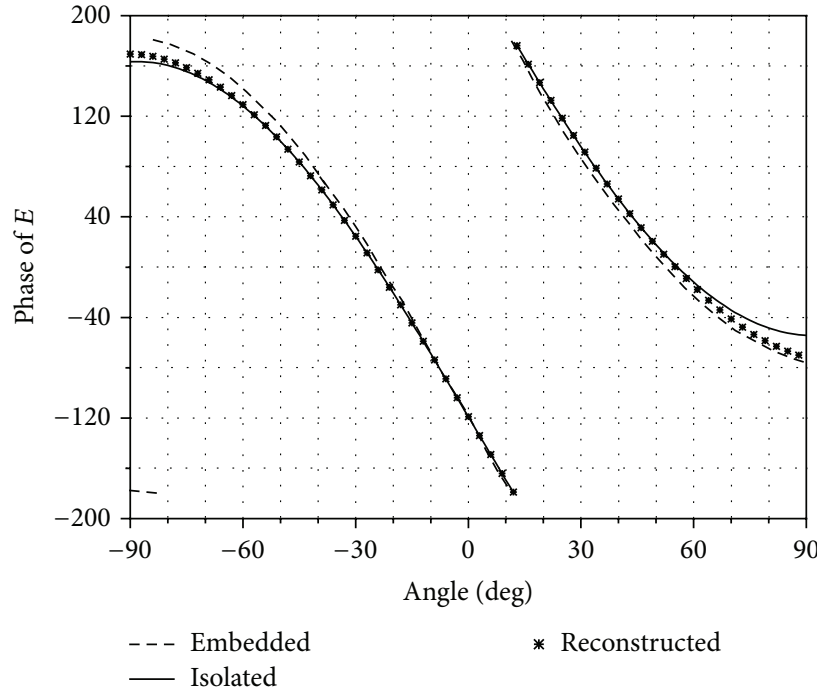

(b)

Figure 7: Magnitude and phase of $\theta$ component of the electric field in three cases for the circularly polarized microstrip array.

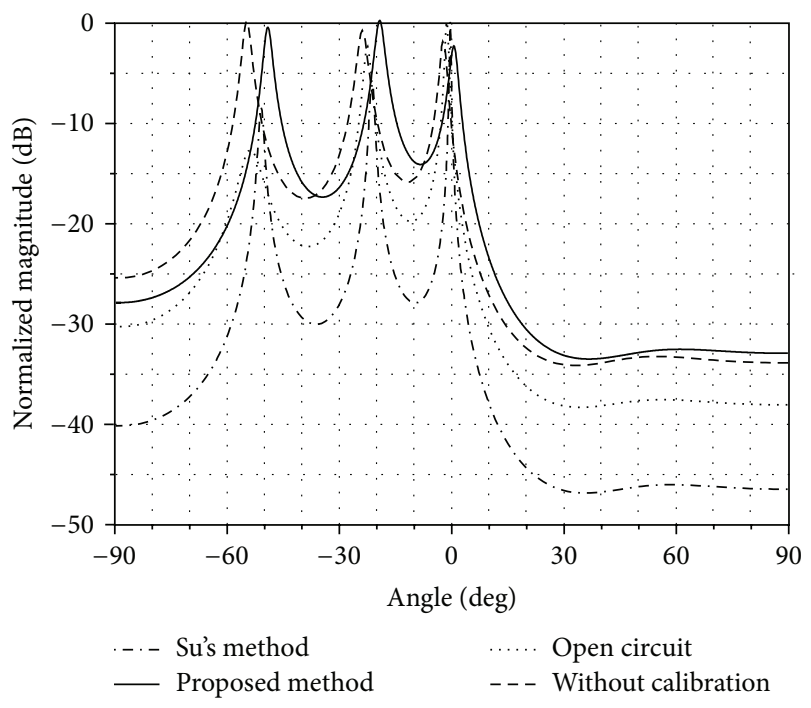

FIGURE 8: Spatial spectrum of DOA estimations for three incident signals employing the circularly polarized microstrip array.

region of the array. Here, the direction sample is represented as $(\theta, \varphi)=\left(10 \alpha^{\circ}, 5 \beta^{\circ}\right)$, where $\alpha=1,2, \ldots, 6$ and $\beta=$ $1,2, \ldots, 71$; that is, the directions with the total of 360 are employed to calculate the calibration matrix.

Performance comparisons of the three calibration methods can be made through the following contour charts of the 2D DOA estimations. As shown in Figure 10, two incident directions can be found due to the proposed method and the open-circuit method, and the proposed method can provide higher DOA estimation accuracy. Additionally, it can be seen that Su's method fails to find the incident directions, which is mainly caused by the extra mutual coupling effect introduced by the transformation between the embedded

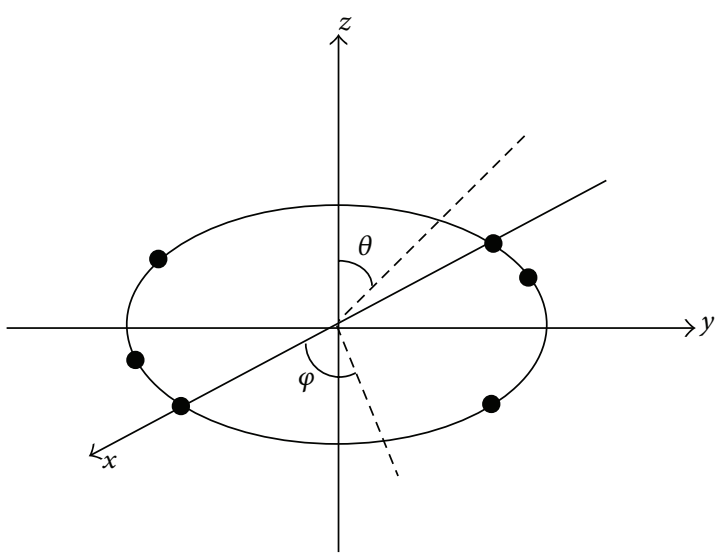

FIgURE 9: Structure of the UCA and the coordinate system.

TABLE 4: Parameters of the incident signals for 2D DOA estimations.

\begin{tabular}{lccc}
\hline Incident signals & $\varphi$ & $\theta$ & SNR $(\mathrm{dB})$ \\
\hline Signal 1 & $20^{\circ}$ & $10^{\circ}$ & 20 \\
Signal 2 & $80^{\circ}$ & $60^{\circ}$ & 20 \\
\hline
\end{tabular}

TABLE 5: Two-dimensional DOA estimation bias for the UCA.

\begin{tabular}{lccc}
\hline \multirow{2}{*}{$(\theta, \varphi)$} & \multicolumn{3}{c}{ DOA estimation bias } \\
& Open circuit & Su's method & Proposed method \\
\hline$\left(10^{\circ}, 20^{\circ}\right)$ & $\left(0^{\circ}, 2.2^{\circ}\right)$ & Failure & $\left(0.4^{\circ}, 0.6^{\circ}\right)$ \\
$\left(60^{\circ}, 80^{\circ}\right)$ & $\left(7.2^{\circ}, 0.8^{\circ}\right)$ & Failure & $\left(0.8^{\circ}, 0^{\circ}\right)$ \\
\hline
\end{tabular}

element patterns and the ideal point source patterns. DOA estimation bias corresponding to Figure 10 is listed in Table 5 which shows the advantages of the proposed method over other two methods. 


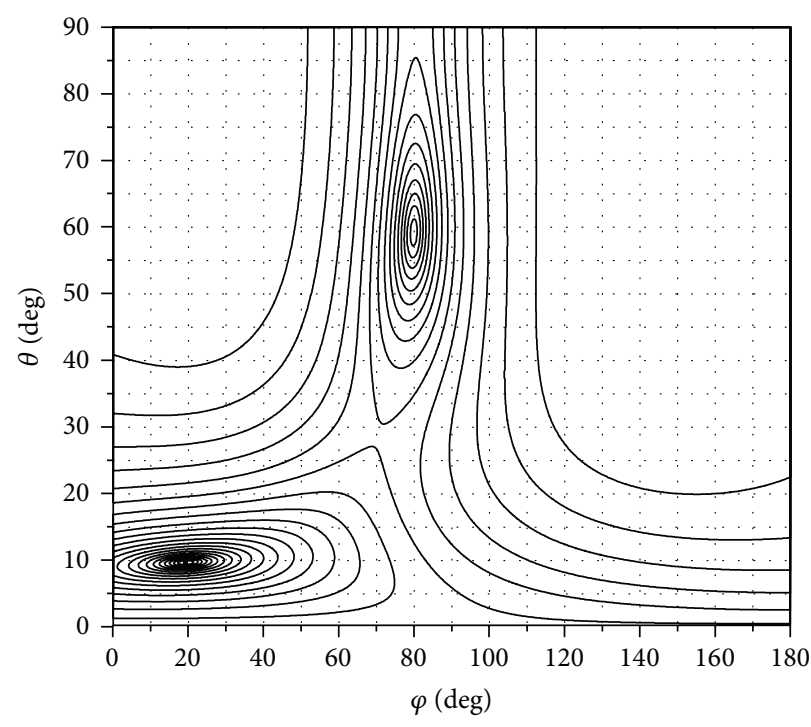

(a)

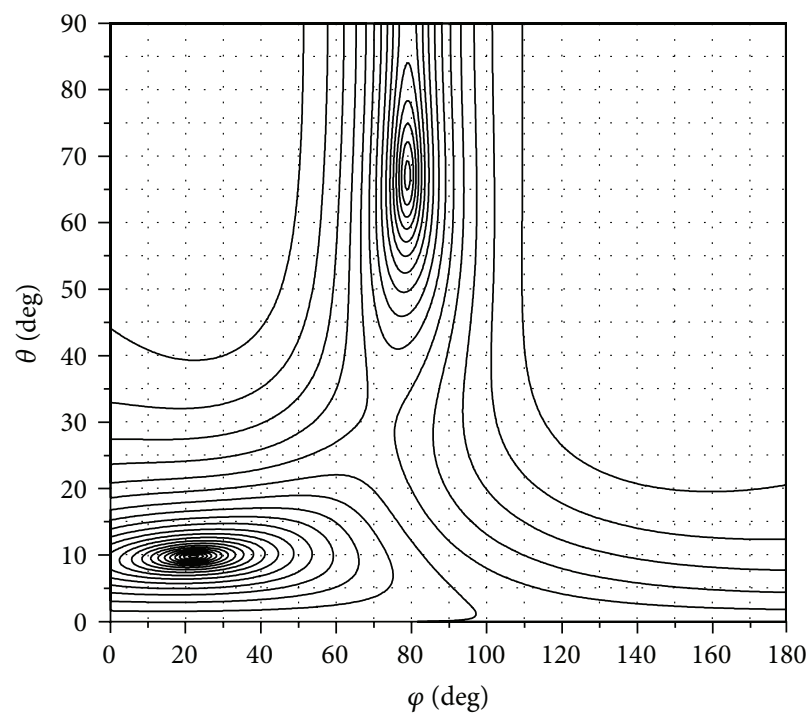

(c)

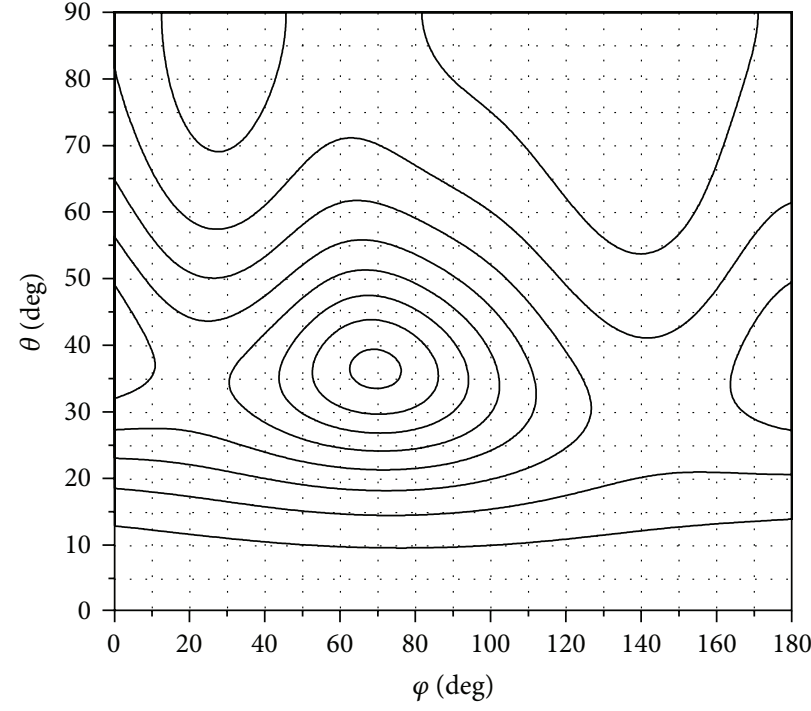

(b)

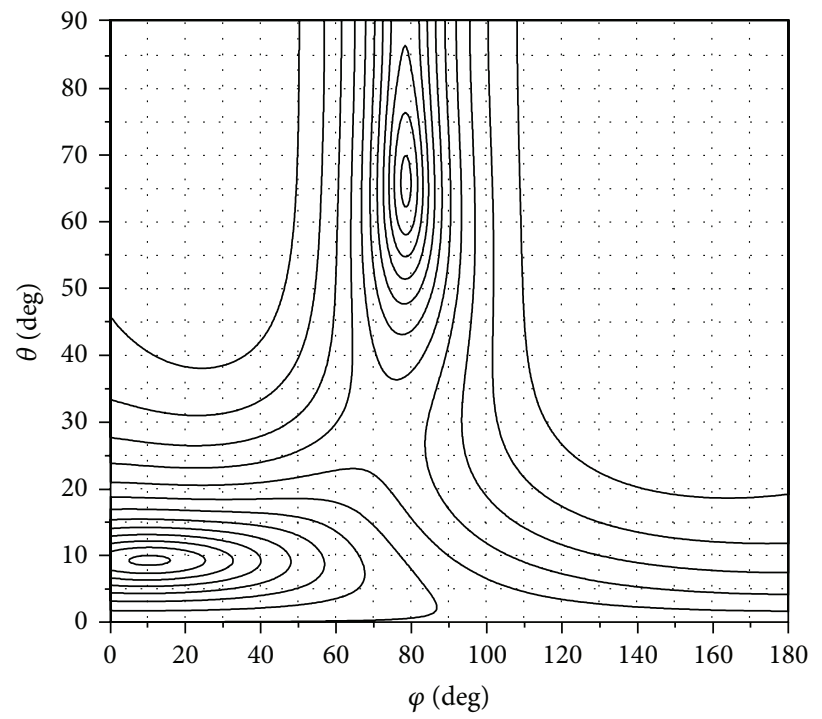

(d)

FIGURE 10: Contour charts of the 2D DOA estimations for (a) the proposed method, (b) Su's method, (c) the open-circuit voltage method, and (d) without calibration.

\section{Conclusion}

In this paper, the element pattern reconstruction method is proposed to calibrate the mutual coupling effect of adaptive antenna arrays. This method is based on the transformation between the embedded element patterns and the isolated element patterns. It is shown that the new method can be employed in microstrip arrays and can provide accurate mutual coupling calibration. Meanwhile, the new method can be used in the linearly polarized microstrip arrays and the circularly polarized microstrip arrays. Owing to the calibration effect of the new method, accurate 1D and 2D DOA estimations can be obtained by employing the MUSIC algorithm in the presence of mutual coupling effect.

\section{Acknowledgments}

This work was supported in part by the "Fundamental Research Funds for the Central Universities" (K5051202012) and the "National Natural Science Foundation of China" (61201019).

\section{References}

[1] R. O. Schmidt, "Multiple emitter location and signal parameter estimation," IEEE Transactions on Antennas and Propagation, vol. 34 , no. 3, pp. 276-280, 1986.

[2] R. Roy and T. Kailath, "ESPRIT-estimation of signal parameters via rotational invariance techniques," IEEE Transactions on 
Acoustics, Speech, and Signal Processing, vol. 37, no. 7, pp. 984995, 1989.

[3] A. G. Jaffer, "Maximum likelihood direction finding of stochastic sources: a separable solution," in Proceedings of the 1988 International Conference on Acoustics, Speech, and Signal Processing, vol. 5, pp. 2893-2896, New York, NY, USA, 1988.

[4] J. A. Cadzow, "A high resolution direction-of-arrival algorithm for narrow-band coherent and incoherent sources," IEEE Transactions on Acoustics, Speech, and Signal Processing, vol. 36, no. 7, pp. 965-979, 1988.

[5] I. J. Gupta and A. A. Ksienski, "Effect of mutual coupling on the performance of adaptive arrays," IEEE Transactions on Antennas and Propagation, vol. 31, no. 5, pp. 785-791, 1983.

[6] R. O. Schmidt, "Multilinear array manifold interpolation," IEEE Transactions on Signal Processing, vol. 40, no. 4, pp. 857-866, 1992.

[7] A. J. Weiss and B. Friedlander, "Manifold interpolation for diversely polarized arrays," IEE Proceedings, vol. 141, no. 1, pp. 19-24, 1994.

[8] A. Manikas, H. R. Karimi, and I. Dacos, "Study of the detection and resolution capabilities of a one-dimensional array of sensors by using differential geometry," IEE Proceedings, vol. 141, no. 2, pp. 83-92, 1994.

[9] T. Su, K. Dandekar, and H. Ling, "Simulation of mutual coupling effect in circular arrays for direction-finding application," Microwave and Optical Technology Letters, vol. 26, no. 5, pp. 331336, 2000.

[10] K. R. Dandekar, H. Ling, and G. Xu, "Experimental study of mutual coupling compensation in smart antenna applications," IEEE Transactions on Wireless Communications, vol. 1, no. 3, pp. 480-487, 2002.

[11] Q. Yuan, Q. Chen, and K. Sawaya, "Accurate DOA estimation using array antenna with arbitrary geometry," IEEE Transactions on Antennas and Propagation, vol. 53, no. 4, pp. 1352-1357, 2005.

[12] H. T. Hui, "Reducing the mutual coupling effect in adaptive nulling using a re-defined mutual impedance," IEEE Microwave and Wireless Components Letters, vol. 12, no. 5, pp. 178-180, 2002.

[13] C. K. E. Lau, R. S. Adve, and T. K. Sarkar, "Minimum norm mutual coupling compensation with applications in direction of arrival estimation," IEEE Transactions on Antennas and Propagation, vol. 52, no. 8, pp. 2034-2041, 2004.

[14] I. Ahmed, W. F. Perger, and S. A. Zekavat, "Effects of ground constituent parameters on array mutual coupling for DOA estimation," International Journal of Antennas and Propagation, vol. 2011, Article ID 425913, 5 pages, 2011.

[15] B. Friedlander and A. J. Weiss, "Direction finding in the presence of mutual coupling," IEEE Transactions on Antennas and Propagation, pp. 273-284, 1991.

[16] M. Lin and L. Yang, "Blind calibration and DOA estimation with uniform circular arrays in the presence of mutual coupling," IEEE Antennas and Wireless Propagation Letters, vol. 5, no. 1, pp. 315-318, 2006.

[17] F. Sellone and A. Serra, "An iterative algorithm for the compensation of toeplitz mutual coupling in UNIFORM and linear arrays," in Proceedings of the IEEE 12th Digital Signal Processing Workshop, pp. 438-443, September 2006.

[18] B. Liao, Z. Zhang, and S. Chan, "DOA estimation and tracking of ULAs with mutual coupling," IEEE Transactions on Aerospace and Electronic Systems, vol. 48, no. 1, pp. 891-905, 2012. 

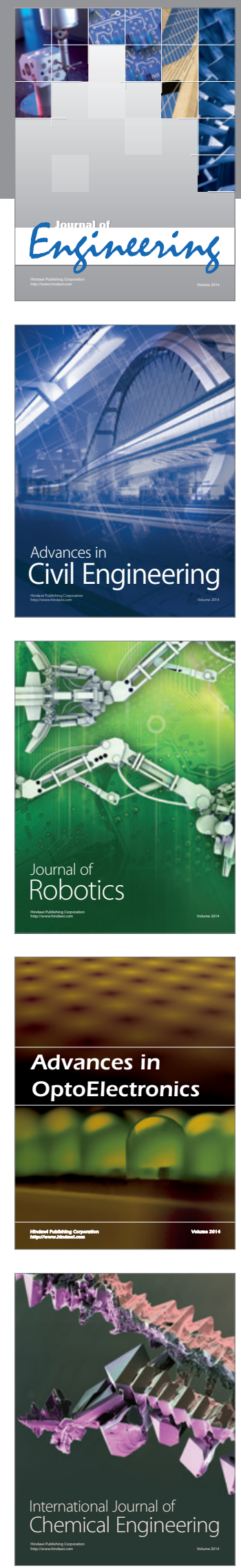

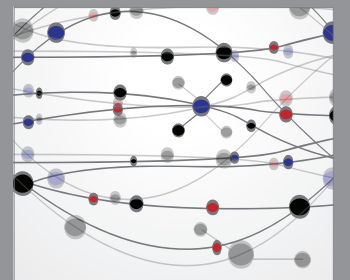

The Scientific World Journal
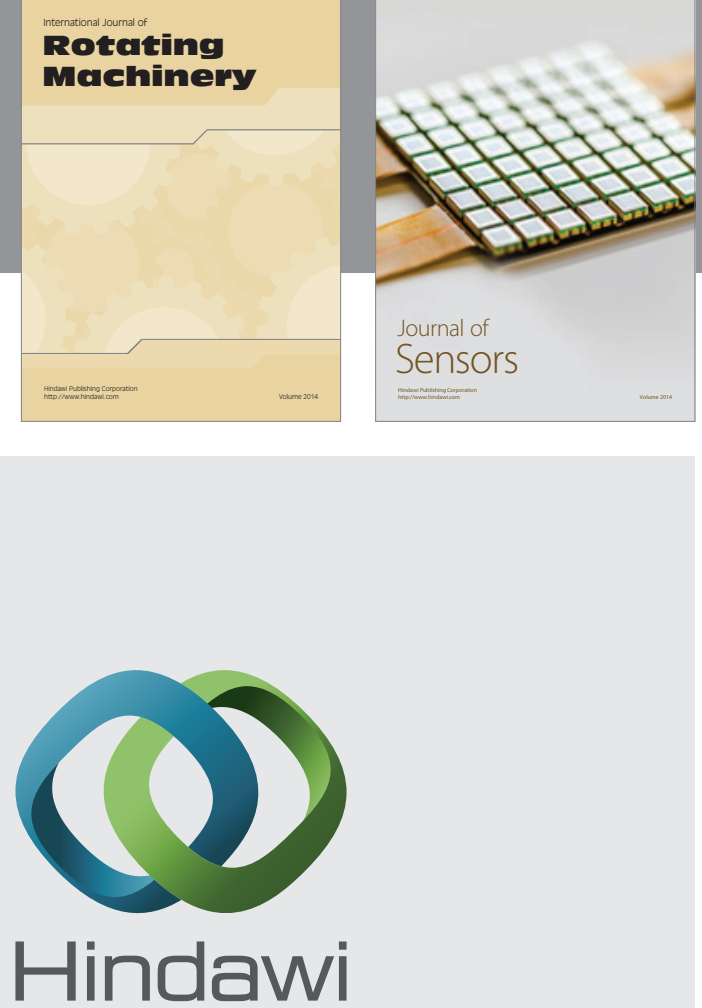

Submit your manuscripts at http://www.hindawi.com
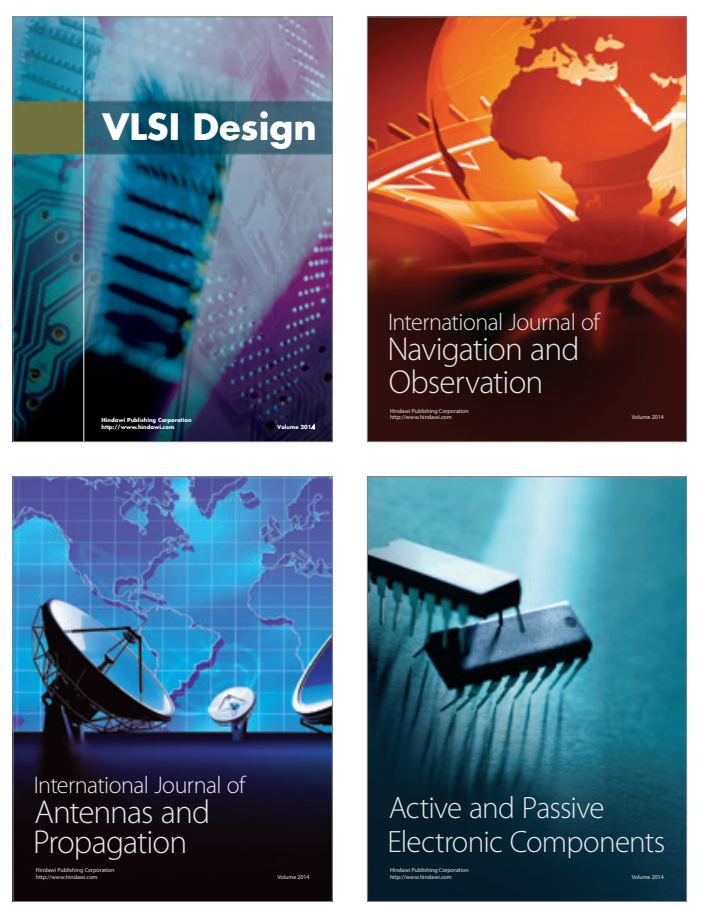
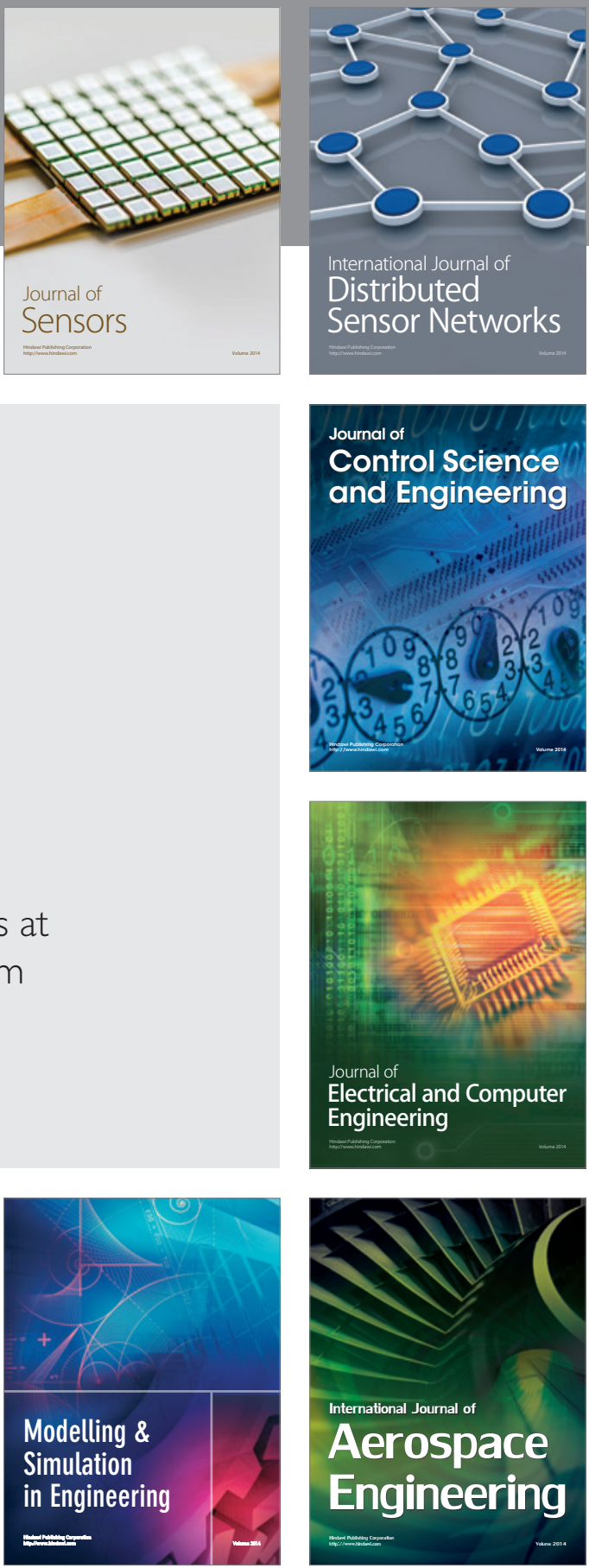

Journal of

Control Science

and Engineering
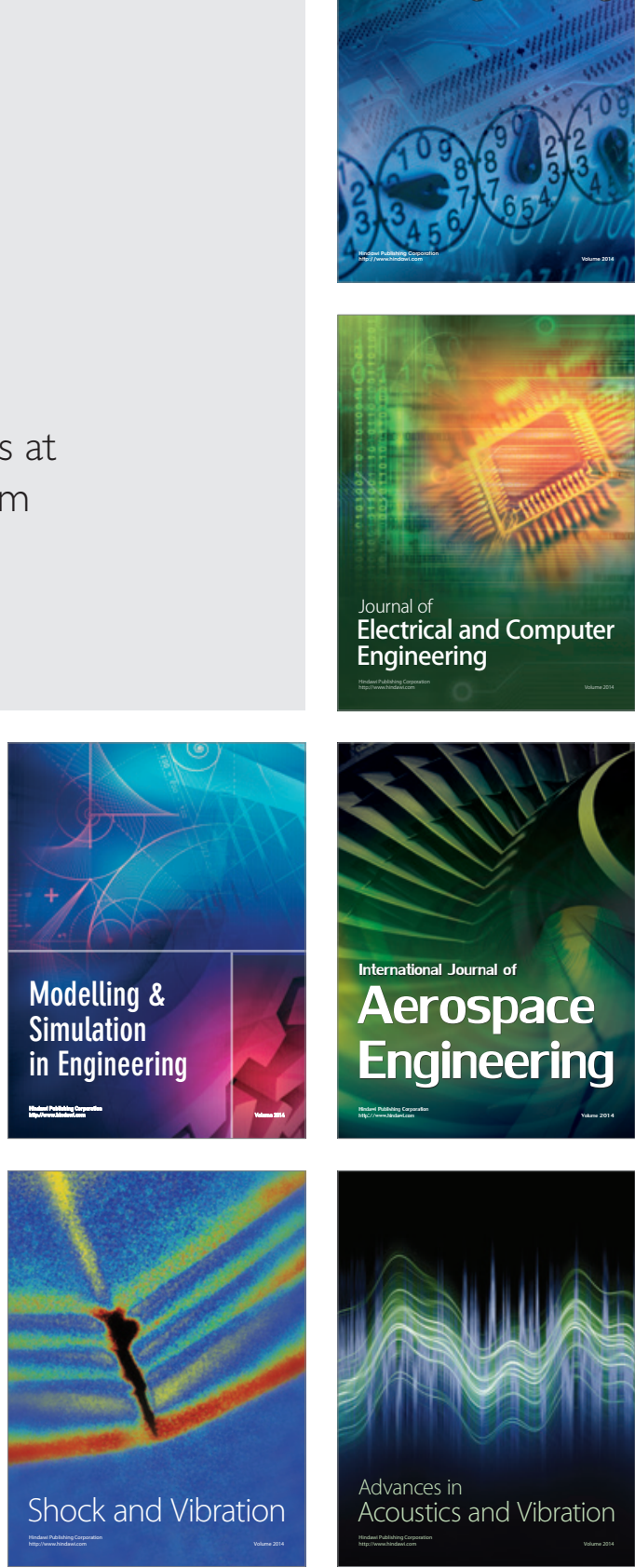